\title{
Advances in Pattern Recognition Techniques, Devices, and Algorithms
}

\author{
Mohammad S. Alam, FELLOW SPIE \\ University of South Alabama \\ Department of Electrical and Computer \\ Engineering \\ 307 North University Boulevard \\ Mobile, Alabama 36688-0002 \\ Mohammad A. Karim, FELLOW SPIE \\ City College of the City University \\ of New York \\ School of Engineering \\ Steinman Hall 142 \\ West 138th Street and Convent Avenue \\ New York, New York 10031
}

The depth and breadth of pattern recognition techniques encompass a rather broad range of research topics. Consequently, the contributors to this special section come not only from the pattern recognition community but also from many other disciplines. Over the last decade tremendous advances have been made in the general area of pattern recognition techniques, devices, and algorithms. The seventeen papers that were finally accepted for this special section encompass the latest trends and advancements made in many different areas of pattern recognition techniques. These techniques include filter based recognition, invariant pattern recognition, joint transform correlator (JTC) based recognition, morphological processing based recognition, multiple sensor based recognition and tracking, wavelet based recognition, and synthetic aperture radar based recognition. These papers summarize the work of 35 researchers from 8 countries.

The first group of three papers addresses the issues pertaining to matched filtering. Jutamulia and Akasura present a simplified theory of the Fourier transform property of lenses based on geometrical optics. This simplified theoretical model is helpful for quick understanding of optical correlators, in particular, and Fourier transform based processors, in general. In the second paper, Khoury, Gianino, and Woods investigate the performance of several optimization algorithms for enhancing correlation performance for severely obscured images in nonoverlapping zero-mean noise. Then Doh, Kim, and Alam propose a new hit-miss transform (HMT) algorithm to detect multiple distorted objects in clutter. Their results show that the proposed algorithm can be used for the recognition of various true class objects in an unknown input scene with only one HMT operation. Thereafter,
Birch, Young, Claret-Tournier, Budgett, and Chatwin demonstrate a complex filter that uses an analog ferroelectric liquid crystal spatial light modulator (AFLC-SLM). The proposed hybrid correlator provides a simpler and more robust solution to implementing a traditional $4 \mathrm{f}$ optical correlator design.

Joint transform correlation is the topic of the next paper, where Iwamoto and Minemoto compare the performances of nonlinear joint transform correlators, the binary subtracted joint transform correlator, and the photorefractive joint transform correlator for input images composed of pure-amplitude, pure-phase, and complex data, respectively. Their study shows that input images with purephase and complex data yield better results than those with pure-amplitude data, for various input images with brighter target scenes. However, input images with pure amplitude data have been found to be more robust for brightness changes of target scenes.

Robust target recognition using wavelet transform based processing is the topic of the following paper. In this paper, Song and Jutamulia describe two new wavelet transforms for noise insensitive edge detection. The proposed algorithm has been found to be especially useful for lead inspection of surface mount devices in the electronics industry.

The next group of papers deals with pattern recognition using adaptive techniques. In the first paper, Billert and Singher introduce a pattern recognition method using multiple filtering. In this technique, a combination of composite filters and a multiple filtering method are used for practical distortion invariant pattern recognition. Then Kwon, Der, and Nasrabadi propose an automatic target recognition system using a multisensor target detection 
technique based on adaptive feature-based fusion algorithm. This technique is especially suitable for detecting low-contrast and blurry targets with relatively low computational complexity. It incorporates multiple imaging sensors in different spectral ranges, and a confidence image is created by adaptively combining the features generated from the multisensor images to obtain accurate potential target locations.

Distortion-invariant pattern recognition is the topic for the next group of papers. In the first paper, Yao, Tan, and Liew propose a novel approach to improve the discrimination of the radial harmonic filter for shift- and scaleinvariant pattern recognition. The approach combines the morphological correlation and radial harmonic expansion for shift- and scale-invariant pattern recognition with improved pattern discrimination. Thereafter, Alsamman and Alam utilize a projection slice synthetic discriminant function based technique for invariant face recognition using fringe-adjusted joint transform correlation. This technique accommodates both in-plane and out-of-plane 3-D distortions for efficient face recognition. Then Yuan and Casasent investigate the pattern recognition of small ships using inverse synthetic aperture radar (ISAR) data. They developed a new weighted-correlation range alignment and weighted multiple-scatterer motion compensation technique for distortion invariant detection of small ships using ISAR data.

The next group of papers deals with the design issues and device/process improvement for pattern recognition applications. In the first paper, Birch, Claret-Tournier, Budgett, Young, and Chatwin outline the design issues associated with hybrid digital-optical hybrid correlators. The authors discuss the design trade-offs associated with optical Fourier transform lens design, mechanical lens housing, and video rate fast Fourier transform digital signal processing (DSP) hardware as well as overall electronic control. Example results using various filters and modulation techniques are described in their paper. Then Khoury, Kane, Gianino, and Woods demonstrate a new type of time-integrative photorefractive device using selfpumped phase conjugation for pattern recognition operations. This device can be used for applications related to time-integrative devices, such as demultiplexors and phase-sensitive detectors. Another new device for angular-multiplexing to frequency-multiplexing conversion is also proposed in this paper. By combining this new device with frequency-demultiplexing, the authors demonstrate a new architecture for retrieving holograms from different holographic storages and for performing various pattern recognition operations on the retrieved information. In the next paper, Khoury, Gianino, and Woods investigate the effect of constant disjoint background illumination on the performance of five optimal correlation filters with obscured inputs. They considered five filters in this study involving the optimization of transmitted matched correlation peak intensity for various parameters such as transmitted input energy, transmitted mismatched energy, and peak-to-correlation energy.

The next paper by Zhang and Karim deals with the application of fractional correlation in fuzzy associative memory (FAM) for pattern recognition applications. They show that the requirement for performing partial fuzzy association in FAM is well met by optical fractional correlation that implements parallel partially space-variant correlation.

Color pattern recognition is the topic of the next paper. In this paper, Corbalan, Millan, and Yzuel demonstrate that color distribution and pattern shape play a significant role in pattern recognition. They propose a color pattern recognition technique using a CIELAB coordinate system, where luminance $\left(\mathrm{L}^{*}\right)$, chroma $\left(\mathrm{C}^{*}\right)$, and hue $\left(\mathrm{h}^{*}\right)$ are used instead of the conventional RGB system. This technique significantly simplifies the recognition process while accommodating illumination variations. In addition, in most cases, the contribution of only two channels $\left(\mathrm{C}^{*}\right.$ and $h^{*}$ ) has been found to be sufficient for avoiding false alarms.

In the last paper of this special section, Yu comments on the legacy of optical information processing with respect to pattern recognition. In this paper, the author discusses the inherent optical complex transformation for information data processing, Fourier and spatial domain processing, composite filtering, 3-D pattern recognition, larger capacity photo-refractive processing, and optical neural computing.

This special section is well balanced and reports on the many ongoing efforts in pattern recognition techniques, devices, and algorithms at different academic, industrial, and government/defense research labs.

We would like to thank the authors for the contribution of their papers, the reviewers for their dedication and timely review, and the Optical Engineering staff for ensuring timely production of this issue.

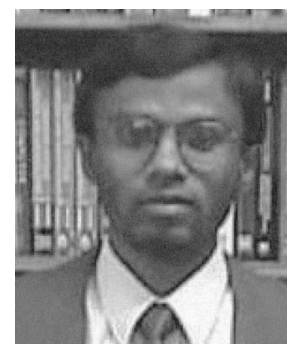

Mohammad S. Alam received his BS and $\mathrm{MS}$ degrees in electrical engineering from Bangladesh University of Engineering and Technology in 1983 and 1985, his MS degree in computer engineering from the Wayne State University in 1989 , and his $\mathrm{PhD}$ degree in electrical engineering from the University of Dayton in 1992. Dr. Alam was on the faculty at Purdue University-Fort Wayne, Indiana, during $1992-1999$ and at the University of Alabama during 1999-2001. He served as a graduate faculty member of both Purdue University and Indiana University during his tenure at Purdue University-Fort Wayne. Dr. Alam joined the University of South Alabama in May 2001 as chair and professor of electrical and computer engineering. His research interests include ultrafast computer architectures and algorithms, digital/ optical signal and image processing, communication systems, pattern recognition, fiber optics, infrared systems, microprocessor applications, and digital system design. He is the principal author of more than 150 published papers, including 75 articles in refereed journals and 8 book chapters (published or to be published by Academic Press, John Wiley and Sons, and Mercel Dekker). He served as the editor of the reference book of selected papers on Real Time Optical Pattern Recognition Using Joint Transform Correlation (SPIE Press, 1999). He is the recipient of the Excellence in Research Award in 1993 and 1997, and the Excellence in Teaching Award in 1995 and 1999, from the School of Engineering and Technology, Purdue University-Fort Wayne. He has also received the 1996 Researcher of the Year Award and the 1998 Teacher of the Year Award from Sigma Xi-the Scientific Honor Society for exceptional accomplishments in research and teaching, and the 1997 Faculty Colloquium on Excellence in Teaching Award from Indiana University for distinguished teaching. Most recently, he received the 1998 Outstanding Engineer Award from Region IV of the IEEE for his outstanding contribution in research, 
teaching, and service to the profession. He has served as a guest editor for four special sections of Optical Engineering. He has served as the principal investigator $(\mathrm{PI})$ or co-PI of many research projects totaling over 6 million dollars. His research work has been supported by federal agencies such as the NSF, FAA, AFOSR, $A R O$, and Wright Patterson Air Force Base as well as by industry such as ITT industry. Dr. Alam has presented over 35 invited papers, seminars, and tutorials at international conferences and research institutions in the USA and abroad. He is a fellow of the Optical Society of America (OSA), a fellow of the International Society for Optical Engineering (SPIE), a senior member of the Institute of Electrical and Electronics Engineers (IEEE), and a member of the American Society for Engineering Education (ASEE). He was the chairman of the Fort Wayne Section of IEEE for 19951996.

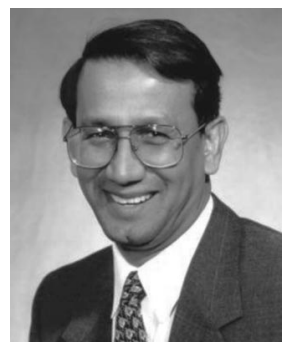

Mohammad A. Karim is dean of engineering of the City College of the City University of New York. He received his BS in physics in 1976 from the University of Dhaka, Bangladesh, and MS degrees in both physics and electrical engineering and $\mathrm{a} P \mathrm{Ph}$ in electrical engineering from the University of Alabama in 1978, 1979, and 1981, respectively. He conducts research in the areas of information processing, pattern recognition, optical computing, displays, and EO systems. Dr. Karim is the author of the books Digital Design: A Pragmatic Approach, Electro-Optical Devices and Systems, Optical Computing: An Introduction, ElectroOptical Displays, and Continuous Signals and Systems Using MATLAB. He has also authored 8 book chapters and over 300 papers. He is the North American editor of Optics \& Laser Technology, an associate editor of the IEEE Transactions on Education and serves on the editorial board of Microwave \& Optical Technology Letters. He has served as guest editor for ten journal special issues. Dr. Karim is a fellow of both the Optical Society of America (OSA) and the Society of Photo-Optical Instrumentation Engineers (SPIE), a senior member of IEEE, and a member of American Society of Engineering Education (ASEE). 